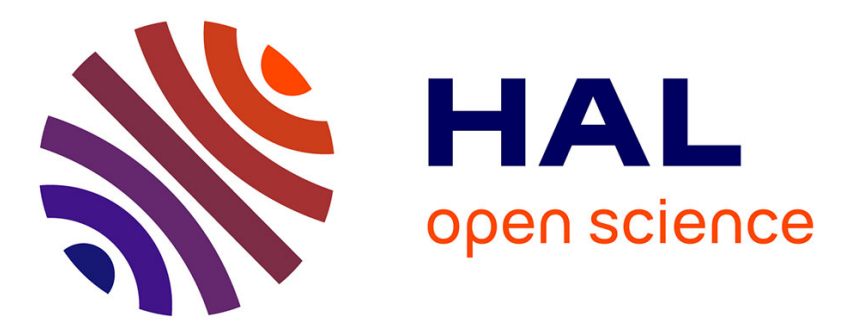

\title{
Wall-Modeled Large-Eddy Simulations of a Multistage High-Pressure Compressor
}

Jérôme de Laborderie, Florent Duchaine, Laurent Gicquel, Stephane Moreau

\section{To cite this version:}

Jérôme de Laborderie, Florent Duchaine, Laurent Gicquel, Stephane Moreau. Wall-Modeled LargeEddy Simulations of a Multistage High-Pressure Compressor. Flow, Turbulence and Combustion, 2020, 10.1007/s10494-019-00094-0 . hal-02571557

\section{HAL Id: hal-02571557 https://hal.science/hal-02571557}

Submitted on 20 May 2020

HAL is a multi-disciplinary open access archive for the deposit and dissemination of scientific research documents, whether they are published or not. The documents may come from teaching and research institutions in France or abroad, or from public or private research centers.
L'archive ouverte pluridisciplinaire HAL, est destinée au dépôt et à la diffusion de documents scientifiques de niveau recherche, publiés ou non, émanant des établissements d'enseignement et de recherche français ou étrangers, des laboratoires publics ou privés. 


\title{
Wall-modeled Large-Eddy Simulations of a Multistage High-Pressure Compressor
}

\author{
J. de Laborderie - F. Duchaine . \\ L. Gicquel · S. Moreau
}

Received: date / Accepted: date

\begin{abstract}
This study aims at evaluating the feasibility and the accuracy of a Wall-Modeled Large Eddy Simulation of an actual aeronautical multistage axial high-pressure compressor. The computational domain is composed of 37 blades and a geometrically complex recirculating cavity. The numerical method TurboAVBP is able to handle such a technical challenge thanks to its unstructured and massively parallel approach as well as dedicated rotor-stator interface treatments. The influence of grid resolution, from less than 100 millions to more than 1 billion of cells, is particularly evaluated. The intermediate grid correctly predicts the global aerodynamic performances up to the lowest mass flow rate regime. In comparing with time-resolved measurements, the finest grid is shown to accurately predict flow within rotors, especially in their tip regions that are critical for performances and stability of the whole compressor.
\end{abstract}

Keywords Turbomachinery · Axial Compressor · Large-Eddy Simulation • High-Performance Computing

\section{Introduction}

Axial compressors represent a critical component of turbofan engines both in terms of efficiency and stability $[8,9]$. Although routinely simulated today in their design phase, the increasing complexity of such flows makes high-fidelity flow predictions

Jerome de Laborderie

Safran Aircraft Engines, 77550 Villaroche, France

E-mail: jerome.de-laborderie@safrangroup.com

Florent Duchaine, Laurent Gicquel

CERFACS, 31100 Toulouse, France

E-mail: florent.duchaine@cerfacs.fr

E-mail: laurent.gicquel@cerfacs.fr

Stephane Moreau

University of Sherbrooke, Sherbrooke J1K 2R1, Qc, Canada

E-mail: stephane.moreau@usherbrooke.ca 
to be expected to provide reliable results with potential breakthroughs in the design of future compressors. However these predictions appear to be challenging as the flow developing in such machines presents high Reynolds and Mach numbers, complex physics and is strongly influenced by practical geometrical details (clearances, cavities, secondary flow systems, etc.). Large Eddy Simulation (LES) has nonetheless been shown to be a promising tool to tackle these challenges $[52,30]$. Indeed, LES resolves a part of the turbulent kinetic energy spectrum corresponding to the largest eddies present in the flow, and models the smallest eddies, that are assumed to follow a universal dissipative behavior [43,45]. Relative to the Unsteady Reynolds-Averaged Navier-Stokes equations (URANS) widely used today in the design of axial compressors, LES has been shown to increase flow prediction reliability, both at design and off-design conditions $[30,14]$. The application of LES to industrial compressors however brings out some challenges [16]. The cost of LES is in the order of $10^{4}$ to $10^{6}$ times the cost of a RANS [15] mainly due to the nearwall grid refinement increase when the Reynolds number increases [53]. Even with the constant increase in computational power, this prevents simulating an entire 360 degrees industrial configuration by use of the academically recommended wallresolved LES approach [43] and such results are to be expected only by the 2035 horizon [16]. Such an estimation however requires a constant and regular increase in CPU power as well as a steady adaptation of existing CFD algorithms which is far from simple and achieved. This inherent limitation is currently identified as the reason why existing LES of compressors concern simplified configurations, such as cascades $[51,49,2]$, isolated blade rows $[19,21]$ or a single stage $[14,26,55]$. Current researches focus on techniques to make LES of compressors affordable, by use of the phase-lagged approach [36] for example and the use of laws of the wall adapted to LES [41]. As no LES of multi-stage axial compressors has yet been reported in the literature, it appears crucial to evaluate the feasibility of such a simulation, in a wall-modeled context since the flow resolution up to the wall is still out of reach and will remain for some time.

Besides the computational power, other technical difficulties need to be treated to effectively simulate a multistage axial compressor with a wall-modeled LES approach. To account for complex geometries such as recirculating cavities, the flow solver should adopt an unstructured formalism, as already pointed out in [57]. Likewise, rotor-stator interfaces are a particular feature of turbomachinery flow simulations. They should be handled with care in compressible LES, as vortical, acoustic and entropy waves should be accurately transferred across these interfaces. Finally numerical schemes and parallelism of the solver should be adapted to such massive simulations. To answer such constraints, the LES solver TurboAVBP has been designed for the high-fidelity prediction of flows in industrial turbomachinery applications [54]. It is based on the coupling of several instances of the reacting LES solver AVBP $[46,1]$. TurboAVBP fulfills the requirements mentioned above for the simulation of a multistage axial compressor. It is indeed a fully unstructured solver. Besides the rotor-stator interface treatment has been thoroughly evaluated in $[54,24]$ and shown to comply with requirements for an accurate LES. Finally the AVBP solver includes high-order numerical schemes already validated and widely used in different applications. It is also a massively parallel solver, with a demonstrated efficient scalability [13]. TurboAVBP has already been applied to fan stages [28,37], a single compressor stage [55], a single turbine stage $[40,39,56]$ and a combustion chamber coupled to a turbine stage [11]. In these cases, comparisons 
with experimental and (U)RANS data highlighted the beneficial contribution of the LES solver even in a wall-modeled context. To further evaluate the capability of this modeling strategy to simulate realistic industrial cases, an aeronautical high-pressure multistage compressor is considered here as it corresponds to the first attempt of such a massive turbomachinery simulation. The configuration is the 3.5 stage axial compressor CREATE, designed by Safran Aircraft Engines and operated at Ecole Centrale de Lyon/LMFA [38]. It is representative of the geometry and flow conditions of the median-rear blocks in a high-pressure compressor of a modern turbofan engine. Several test campaigns using advanced measurement techniques allowed an accurate characterization of the flow, making possible detailed evaluations of numerical methods for turbomachinery. For confidentiality reasons, quantities are normalized in this study.

CREATE has already been extensively studied numerically, for numerical method evaluations as well as for investigation of physical phenomena. URANS simulations of CREATE have been assessed against experimental data [38,5] allowing to study a casing treatment for control of tip leakage flows [17]. Crevel et al. [6] and Schreiber et al. [48] performed URANS simulations at low mass flow rate for instability investigation, even simulating a full surge cycle [7]. However some limitations in the application of URANS to axial compressors could be evidenced. For instance, the flow dynamics around rotor blades tips represents a challenge for turbulence models and might be poorly predicted [33]. The lack of accuracy in reaching the lowest mass flow rate regime is also a typical weakness for RANS approaches [47]. High-fidelity methods such as the Detached Eddy Simulation (DES) have been employed to study the tip flow in the first rotor of CREATE [44]. Preliminary results obtained with TurboAVBP at nominal conditions have also been presented in [25], as well as a study at off-design conditions [23]. The present paper aims at evaluating more accurately these simulations at nominal conditions.

The objectives of this study consist in the feasibility demonstration of a wallmodeled LES of a multistage high-pressure compressor, as well as in an accurate evaluation of the predictions using a large experimental database. The influence of grid resolution is particularly studied, from global performance predictions to more detailed flow physics. The experimental test rig, measurement techniques and essential features of compressor aerodynamics are briefly introduced in Sec. 2. The numerical method and the computational domain are then presented in Sec. 3. The simulated flow fields are first evaluated in terms of global performances and radial profiles in Sec. 4. Taking profit of available unsteady experimental data, simulations are further evaluated downstream rotors in Sec. 5 and in the tip region of first rotor in Sec. 6. Finally loss generation in the entire compressor is assessed in Sec. 7.

\section{Multistage axial compressor}

This section presents the CREATE compressor and introduces some basic of compressor flow physics. 


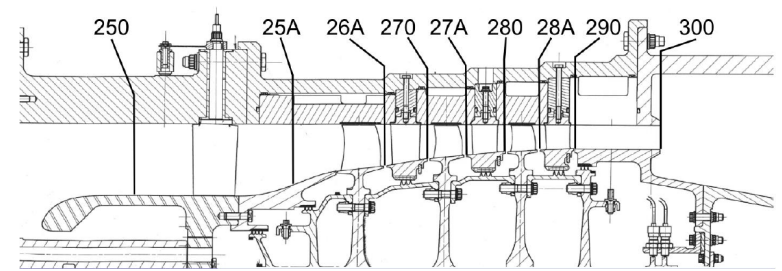

Fig. 1 Meridian cut of the compressor CREATE, from [38].

Table 1 Number of blades in each row of CREATE.

\begin{tabular}{cccccccc}
\hline Row & IGV & R1 & S1 & R2 & S2 & R3 & S3 \\
\hline For $2 \pi$ & 32 & 64 & 96 & 80 & 112 & 80 & 128 \\
For $2 \pi / 16$ & 2 & 4 & 6 & 5 & 7 & 5 & 8 \\
\hline
\end{tabular}

\subsection{Experimental facility and measurement techniques}

The multi-stage research compressor CREATE (Compresseur de Recherche pour l'Etude des effets Aerodynamiques et TEchnologiques), designed by Safran Aicraft Engines and operated at LMFA (Ecole Centrale de Lyon, France), is the machine chosen for the present study. The configuration corresponds to the CREATE2bis geometry. CREATE is a high-pressure high-speed 3.5 stage axial compressor, representative of high-pressure compressor median-rear blocks in modern turbofan engines. Figure 1 presents a view of the meridian cut of the compressor, showing a variable Inlet Guide Vane (IGV), three rotors (R1, R2, R3) and three stators (S1, $\mathrm{S} 2, \mathrm{~S} 2$ ). The number of blades per row, noted in Table 1, has been chosen in the design process so that the compressor has a natural circumferential periodicity of $2 \pi / 16$. The casing outer diameter is constant throughout the machine. The design shaft speed is $11543 \mathrm{rev} . / \mathrm{min}$., inducing a 0.92 inlet Mach number at the tip of $\mathrm{R} 1$. Hence the flow is slightly transonic in the first rotor and fully subsonic in the other rows. The Reynolds number based on the blade chord at the tip of R1 is around $10^{6}$.

Several measurement techniques have been developed and employed to characterize the flow in CREATE. This compressor being installed within an open-loop facility, the mass flow rate is measured with a Venturi nozzle located in the exhaust part of the rig. For the other global performance measurements, a thermocouple and a Kiel probe located at the inlet (section 250 in Fig. 1) provide the total temperature and pressure respectively. At the outlet (section 300 in Fig. 1), total pressure and temperature probe rakes measure the flow at six circumferential and five radial positions. The uncertainties on these global quantities have been quantified in Ottavy et al. [38]. These were found to be rather independent of the operating conditions [3] and are $\pm 0.35 \%$ and $\pm 0.07 \%$ on mass flow rate and pressure ratio respectively. In addition to the global performance characterizations, steady measurements of total and static pressure, total temperature and flow angle have been performed with 5-hole probes in all the inter-row sections shown in Fig. 1, from 26A to 290. Note that the flow unsteadiness has also been characterized by fast pressure probes [31,32] and two-component Laser Doppler Anemometry (LDA) in the inter-row sections [3]. LDA being a non-intrusive tech- 


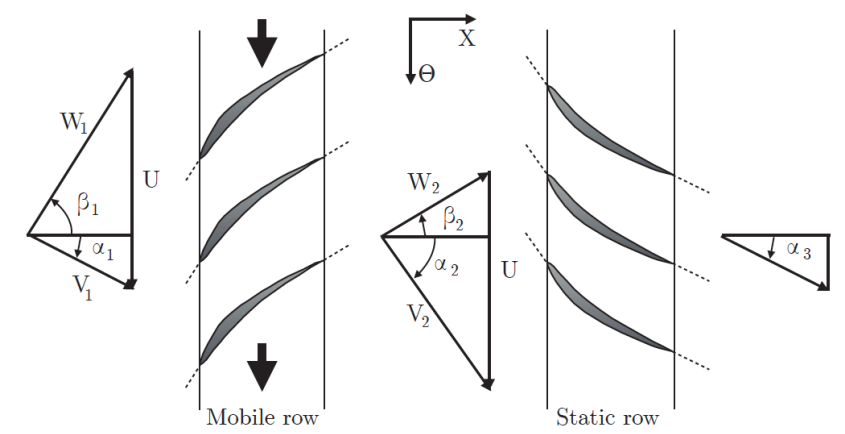

Fig. 2 Sketch of velocity triangles in a single axial compressor stage at a given radial location in the duct, from [35].

nique, it could be used to measure the flow within rotor blade channels, especially near the blade tips [3]. Finally fast pressure transducers flush mounted within the tip casing are also a way to characterize the flow in the tip region of rotors $[3,4]$.

\subsection{Elements of axial compressor aerodynamics}

Before the analysis of the results presented in this paper, some concepts and definitions regarding compressor aerodynamics need to be introduced. For a given rotational speed, increasing the outlet pressure leads a compressor to produce more work on the flow. The resulting mass flow rate decreases up to a minimal value, beyond which the compressor surges and flow reverses. The accurate prediction of the stable operating conditions at the lowest mass flow rate is critical for design and it is presently challenging for any numerical methods.

Figure 2 represents a 2-D cut at a radius $r$ of an axial compressor stage composed of a rotor and a stator. The rotor is moving from the top to the bottom with the velocity $\mathbf{U}=r \boldsymbol{\Omega}$, where $\boldsymbol{\Omega}$ is the rotational speed of the compressor. The absolute velocities in the static reference frame are denoted as $\mathbf{V}$, and the velocities in the moving reference frame, called relative velocities, are written as W. By definition, these velocities are linked with the speed triangle relation:

$$
\mathbf{V}=\mathbf{W}+\mathbf{U}
$$

The absolute flow angle, denoted $\alpha$, is thus the angle between the absolute velocity $\mathbf{V}$ and the axis $x$ of the machine. The flow turning represents the deviation performed by a blade profile to the flow. It is $\beta_{1}-\beta_{2}$ for a moving blade, and $\alpha_{2}-\alpha_{3}$ for a static vane.

Flow viscosity is responsible for losses that drastically impact performances of compressors. These losses, identified in [10] for instance, should be accurately predicted by the numerical simulation. Two main losses phenomena are presented here. Boundary layers on pressure (PS) and suction (SS) sides of a rotor blade profile merge at its trailing edge to form a wake. The latter contains lower energy fluid and higher turbulence intensity than the flow outside the wake. As represented in Fig. 3(a), this wake interacts with a downstream vane profile. This interaction 


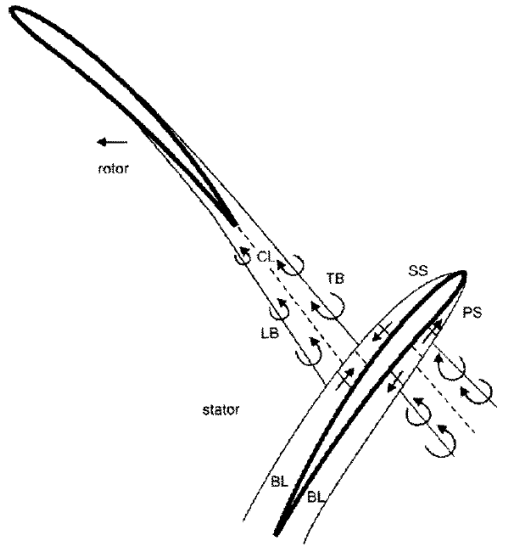

(a) Sketch of rotor wake interaction with stator vane boundary layer, from [29].

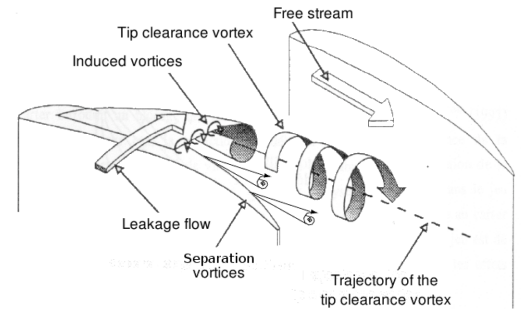

(b) Sketch of typical flow structures in the tip region of a compressor rotor blade, from [3].

Fig. 3 Two significant losses phenomena in an axial compressor.

may modify its suction side boundary layer. Blade wakes and their interactions represent a major part of losses in compressors. Additionally, a clearance between the rotor blade tips and the casing wall is required to ensure mechanical integrity of the blades. Due to the positive pressure gradient between PS and SS, a tipleakage flow appears within the clearance and exits as a jet in the blade passage. The interaction of this jet with the main flow generates the so-called tip clearance vortex (TCV) that develops as represented in Fig. 3(b). Tip separation vortices (TSV) can also be present along the downstream part of the blade. These phenomena interact with the casing boundary layer and possible upstream wakes, creating a complex flow region at the tip of a compressor rotor that is responsible for a large part of losses [10]. These two phenomena contain significant turbulent structures that LES is expected to capture accurately. These losses phenomena are particularly studied in this paper.

\section{Numerical set-up}

3.1 LES solver for turbomachinery flows

The strategy retained for TurboAVBP relies on the external coupling of separate instances of the AVBP LES solver [46,1]. Within each AVBP instance, the filtered unsteady compressible Navier-Stokes equations are solved on unstructured grids. These equations describing the mass, momentum and energy conservations, can be written in the absolute reference frame as:

$$
\frac{\partial \mathbf{A}}{\partial t}+\nabla \cdot \mathcal{F}=0
$$

A contains the conservative variables $(\rho, \rho \mathbf{V}, \rho E)$ where $\rho$ is the fluid density, $\mathbf{V}$ is the velocity vector and $E$ is the total energy of the fluid. $\mathcal{F}$ is the flux tensor, 
decomposed into the convective part: $\mathcal{F}^{c}(\mathbf{A})$ and the viscous part: $\mathcal{F}^{v}(\mathbf{A}, \nabla \mathbf{A})$. This viscous flux contains the contributions of the unresolved flow scales whenever derived in the context of LES. For non-reacting and single-species flows, these contributions are modeled under the form of the Sub-Grid Scale (SGS) stress tensor, using the Boussinesq assumption [43]:

$$
\tau_{i j}^{t}=2 \rho \nu_{t}\left(S_{i j}-\frac{1}{3} \delta_{i j} S_{l l}\right)
$$

where $S_{i j}$ is the resolved strain rate tensor and $\nu_{t}$ is the SGS turbulent viscosity to be modeled. To be consistent between the presented simulations, only a single SGS model is employed, namely the Smagorinsky sub-grid scale model [50], for which the turbulent viscosity is:

$$
\nu_{t}=\left(C_{S} \Delta\right)^{2} \sqrt{2\left(S_{i j} S_{i j}\right)},
$$

where $\Delta$ is the characteristic length of the grid and $C_{S}=0.18$ the Smagorinsky constant. For consistency and cost reasons, the finite volume Lax-Wendroff (LW) scheme, second-order accurate in space and time [27], is used for all simulations.

In moving domains, the mesh movement is performed with the ALE method [34] and the equations are solved in the absolute reference frame. The external coupling of several AVBP instances is performed with the OpenPALM coupler [12], co-developed by ONERA and CERFACS. Each computational domain with its own grid velocity is attributed to a dedicated instance. The transfer of information between these domains is achieved with the overset grid method MISCOG (Multi Instance Solver Coupled through Overlapping Grids), proposed and detailed by Wang et al. [54]. The principle consists in the exchange, at each time step, of the conservative variables at nodes belonging to cells that overlap around the interfaces. Since the overlapping cells are not coincident, data are interpolated at each exchange. MISCOG was originally developed with a spatially second order accurate interpolation [54] and has recently been extended with a third order accurate interpolation, using nodal gradients and the Hermite polynomial technique [24]. As recommended, this high-order interpolation is used in all simulations presented. Note that the overset grid method, unless specifically constructed to do so, is not fully conservative. The conservation properties of MISCOG on mass flow and energy have already been addressed in [54]. To limit conservation variation at each interface, similar sizes for overlapping cells are recommended. Using this condition, the relative mass and energy errors across each interface are found to be less than $0.1 \%$ and $0.2 \%$ respectively in all the simulations.

\subsection{Computational domain}

The computational domain for the TurboAVBP simulations, shown in Fig. 4, corresponds to an azimuthal sector of one sixteenth of the machine, following its natural periodicity (see Tab. 1). This choice forces the periodicity of the flow at the lateral boundaries, which naturally infers a limitation to simulate the actual flow developing in the machine. This azimuthal limitation is driven by the huge computational cost that a 360 degree simulation would represent and the natural geometrical periodicity of the machine. The TurboAVBP strategy consists here 


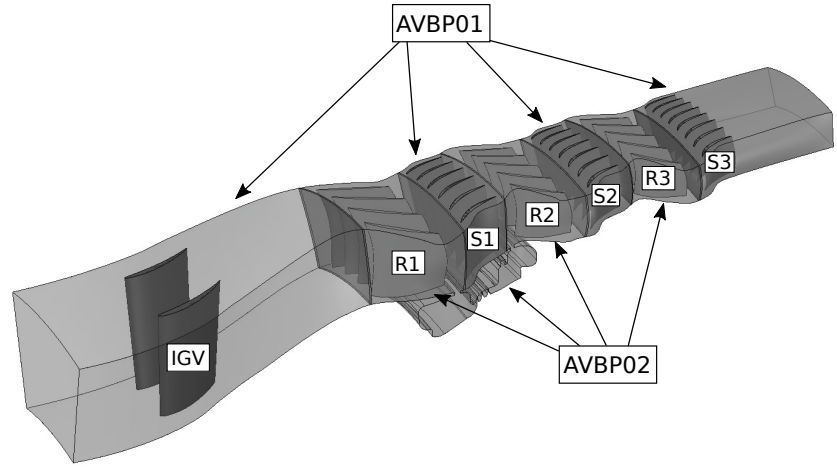

Fig. 4 Computational domain for TurboAVBP.

in attributing one instance (AVBP01) to the fixed domain containing the stator rows, and the other instance (AVBP02) to the moving domain containing the rotor rows, since all the rows rotate at the same speed (Fig. 4). Several technological effects are accounted for in the simulations, including the actual tip clearances between the three rotors and the casing, as well as the recirculating cavity with a labyrinth seal below the first stator. For practical reasons, this cavity is embedded within the moving instance and is attached to the R1 and R2 domains. A fixed wall boundary condition is imposed to the top wall of the cavity. As shown in Fig. 4, 6 overlapping MISCOG interfaces are present within the computational domain, between each rotor and stator rows. Note that it has recently been shown that successively adding MISCOG interfaces does not deteriorate the global properties of the convective scheme [25].

The inlet of the domain corresponds to plane 250 in Fig. 1, located 1.3 axial Inlet Guide Vane (IGV) chord upstream of the IGV, where the measured mean total pressure and total temperature radial profiles are imposed uniformly in the azimuthal direction. No turbulence is injected through this inlet. Plane 300 is the outlet of the domain, located 2.3 axial S3 vane chords downstream of the third stator, where a target static pressure is imposed. To ensure non reflection of acoustic waves at the inlet and at the outlet of the domain, Navier-Stokes Characteristic Boundary Conditions (NSCBC) are employed [42]. It has recently been shown that a characteristic outlet boundary condition including transverse terms [18] is particularly suited for turbomachinery flows since it allows the radial equilibrium to be naturally taken into account around the target static pressure value [22]. An adiabatic wall-law condition is imposed to the whole set of walls present in the simulation, i.e. blades, hub, tip casings and cavity.

\subsection{Grids generation}

Three fully unstructured grids have been generated for the computational domain shown in Fig. 4. Their main features are gathered in Tab. 2. M1 is a fully tetrahedral grid whereas several layers of prisms on all the blades and vanes are present in M2 and M3. The prismatic cells allow to decrease the distance to the wall of the first node inside the flow while limiting the additional amount of cells com- 


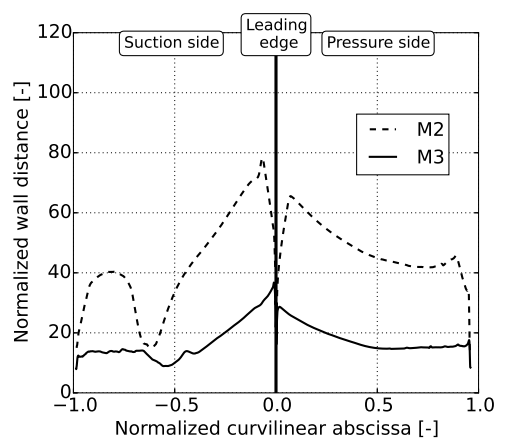

(a) R1 blade.

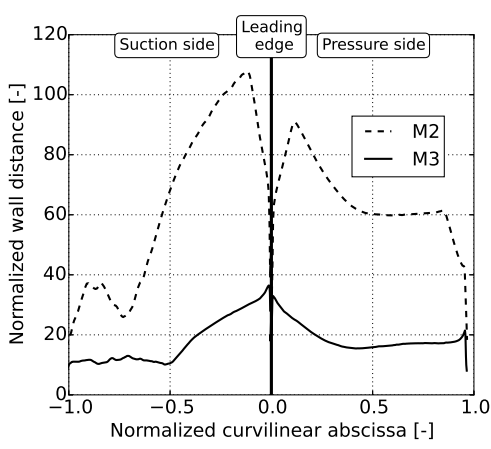

(b) S3 vane.

Fig. 5 Time-averaged normal distance to the wall $\mathrm{y}^{+}$, at midspan of blades.

Table 2 Main properties of the grids. Performances measured on Intel Haswell 2.6 GHz processors.

\begin{tabular}{lccc}
\hline Grids & M1 & M2 & M3 \\
\hline Nb. stator cells $\left(\times 10^{6}\right)$ & 88 & 194 & 1032 \\
Nb. prism layers on blades & 0 & 3 & 10 \\
Nb. cell layers in rotor tip gaps & 3 & 6 & 20 \\
Max. y ${ }^{+}$on blades & 500 & 100 & 40 \\
Grid cut-off frequency $(\mathrm{kHz})$ & 214 & 256 & 510 \\
Time step (s) & $4 \times 10^{-8}$ & $2 \times 10^{-8}$ & $5.4 \times 10^{-9}$ \\
CPU cost per revolution $(\mathrm{kh})$ & 17 & 165 & 2020 \\
\hline
\end{tabular}

pared to a fully tetrahedral grid. Note that with more than one billion of cells, the M3 grid agrees with the expected order of magnitude of a LES grid for industrial compressors at $\mathrm{Re} \approx 10^{6}[52]$. The time-averaged dimensionless wall distance $\mathrm{y}^{+}$ is shown in Fig. 5 for one blade and one vane, for the M2 and M3 grids. For M3, the maximum $\mathrm{y}^{+}$value is less than 40 over the whole set of blades, and less than 100 for M2. This is in agreement with recommendations for wall-modeled LES [45, 16]. The order of magnitude of $\mathrm{y}^{+}$in M1 is around 500.

While generating such grids, particular attention has been paid to the size of the triangle faces of the prisms on all the blade surfaces. The equivalent dimensions in the longitudinal and transverse directions are $\Delta \mathrm{x}^{+}=\Delta \mathrm{z}^{+}=3 \Delta \mathrm{y}^{+}$for M2, and $\Delta \mathrm{x}^{+}=\Delta \mathrm{z}^{+}=6 \Delta \mathrm{y}^{+}$for M3. Hub and casing walls are meshed with tetrahedral cells, giving a $\mathrm{y}^{+}$value around 100 for M3. Note that for this most refined mesh, a particular effort has been devoted to properly resolve the gaps at the tip of the rotor blades, with 20 layers of cells (see Tab. 2). It is indeed crucial to accurately resolve the flow in these regions as it drives the performances and the stability of the whole compressor [16]. Finally the cut-off frequencies of the grids presented in Tab. 2 are large enough to ensure a proper grid resolution of the unsteady phenomena studied. Indeed the blade passing frequency (BPF) of R1 is $12.3 \mathrm{kHz}$ for instance and is well below grid limits. 
3.4 Computational cost and convergence of the simulations

The increasing amount of degrees of freedom from grids M1 to M3 naturally impacts the computational cost mainly by the time step imposed by the explicit formulation of the solver and the constant value of the CFL number for each grid $(\mathrm{CFL}=0.7)$. As shown in Tab. 2, the CPU cost increases by one order of magnitude from M1 to M2 and from M2 to M3, as measured on Intel Haswell 2.6 $\mathrm{GHz}$ processors. The affordable cost for M1 and M2 grids allows the simulation of several revolutions. Based on modifications of a boundary condition, it has been observed that the transient period of the machine can be as long as two rotor revolutions. This corresponds to the time for the flow to reach an equilibrium state between the imposed inlet total pressure, the adverse outlet static pressure, the work brought by the rotors as well as the physical and numerical losses within the domain. The criterion retained for flow convergence in the compressor is in what follows based on the mass flow rate. Following the transient period, it has been verified that the mass flow rate variation is indeed less than $0.1 \%$ between two successive revolutions at each operating condition for M1 and M2. The corresponding simulations are thus considered converged in terms of mass flow rate, and statistics are recorded during two successive revolutions. Due to the large computational cost of M3, its initial solution results from the interpolation of the M2 converged flow field. Two revolutions of M3 have then been simulated, during which it has been observed that the mean temporal evolution of the outlet mass flow rate varied by less than $0.3 \%$ over the last revolution so flow statistics are extracted from this period.

\section{Evaluation of mean flow fields}

\subsection{Global performances}

Figure 6 represents the performance curve of CREATE at nominal speed in terms of total pressure ratio as a function of mass flow rate as introduced in Sec. 2.2. The values are normalized by the experimental quantities at the nominal point, denoted A in the figure. The experimental data are shown with their uncertainties evaluated in [38]. LES results, represented with circles for M1, squares for M2 and triangle for M3, are post-processed consistently with measured data. Namely the total pressure ratio is defined as:

$$
\Pi=\frac{\overline{P_{t, o}}}{P_{t, i}},
$$

where $P_{t, i}$ is the time-averaged total pressure in plane 250 at one point at midheight of the duct. In the test rig, the output total pressure $\overline{P_{t, o}}$ is the arithmetic average of measurements over 30 probes located in plane 300, equally distributed on 6 radial rakes over the full annulus. For LES, $\overline{P_{t, o}}$ is defined as:

$$
\overline{P_{t, o}}=\frac{1}{15} \sum_{k=1}^{15} P_{t, o, k},
$$




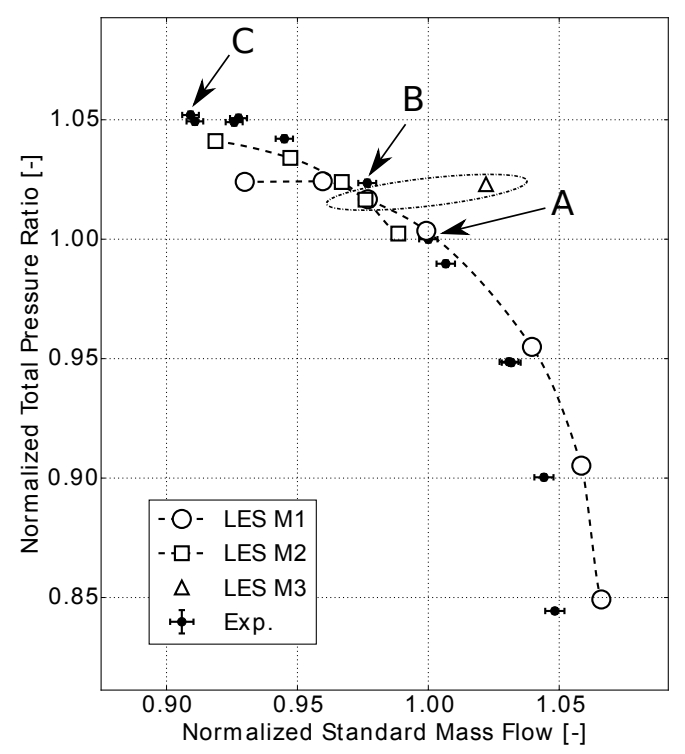

Fig. 6 Aerodynamic performance curve of CREATE. Comparison of LES predictions with experimental data.

where the 15 numerical probes $k$ are distributed along three radial lines over the $2 \pi / 16$ sector. The radial locations in the computation match the positions of experimental probes. In addition to the uncertainties in measurement chains, Schreiber et al. [48] have recently quantified some discrepancies between experimental and existing numerical studies of CREATE. First, the geometry of the computational domain is simplified as it accounts neither for leakages at hub and casing between each row, except below S1, nor for hub and tip clearances of IGVs. Second, the eight struts supporting the hub upstream of plane 250 (see Fig. 1) are not included in the domain. The estimated offsets created by these discrepancies are estimated at $3.8 \%$ on the mass flow rate and 0.1 total pressure ratio point. Using these estimations, the LES predictions are found within the correct range relatively to the measurements.

As explained in Sec. 3.4, the computational cost for simulations on M1 and M2 grids is affordable, hence allowing the simulation of several operating conditions. These points in Fig. 6 are obtained by varying the pressure imposed at the outlet. Each point corresponds to the simulation of at least four revolutions to ensure the mass flow rate stabilization. It can be noticed that shapes and levels of both M1 and M2 curves are rather close to the experimental iso-speed line up to a normalized mass flow of 0.96 . Further reducing mass flow rate shows that M2 better predicts the measured operating points than M1, including the last stable point denoted C. The grid refinement effort from M1 to M2 mainly concerns near wall regions of blades and tip clearances of rotors. An improved prediction of the flow dynamics and losses is thus expected in these zones that are critical for global performance prediction. The lowest mass flow rate operating point for M1 has been obtained 


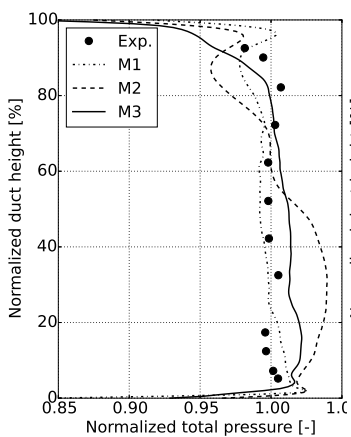

(a) Total pressure.

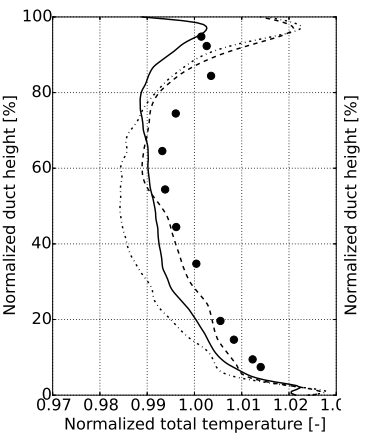

(b) Total temperature.

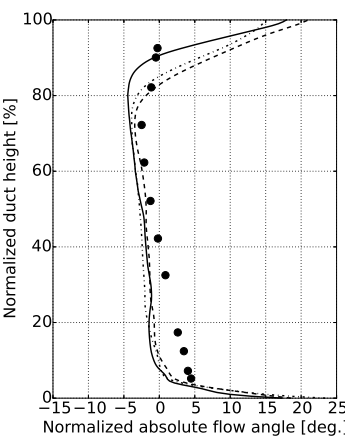

(c) Absolute flow angle.

Fig. 7 Radial distribution of time and azimuthally averaged quantities at section 26A downstream of R1.

for a variation of $3.2 \%$ of back pressure relatively to nominal conditions. It has furthermore been verified that an increase of $0.3 \%$ of back pressure from this M1 last point leads to mass flow reversal, or numerical surge. For M2 grid, the lowest mass flow rate conditions correspond to a variation of $5.3 \%$ of outlet pressure with respect to nominal conditions. The grid refinement is thus crucial to get stable operating conditions at lower mass flow rates, approaching the experimental stability limit. As detailed in Sec. 3.4, only one operating point has been simulated with the M3 grid. The resulting mass flow rate is around $4 \%$ higher than for M1 and M2 at this pressure ratio value. This slight gap is caused by the mass flow rate convergence, not yet strictly reached.

Experimental point B in Fig. 6 corresponds to operating conditions for which the database used in this study is available. In the following, only the three simulations circled in the figure will be considered, since one of the objectives of the paper is to evaluate LES with respect to measurements close to the nominal regime of the compressor.

\subsection{Evolution of radial profiles along the compressor}

Following the LES evaluation with 0-D quantities, this section focuses on the comparison of radial profiles. The latter are experimentally obtained with 5 -hole probes that can be placed in each inter-row section, at several radial locations. These sensors provide time-averaged quantities on a $2 \pi / 16$ sector, that are later circumferentially averaged, using the local mass flow rate weighting $\rho u_{x}$ for total values. Figures 7 to 12 compare these experimental data to the time and circumferentially averaged data from LES, using the same procedure as for measured data. Following the experimental procedure, the numerical data are weighted by the local mass flow rate $\rho u_{x}$ for the averaging. The total pressure and temperature values are normalized by the radially averaged experimental data at each section and the absolute flow angle, introduced in Sec. 2.2, is normalized by removing the averaged experimental angle. 


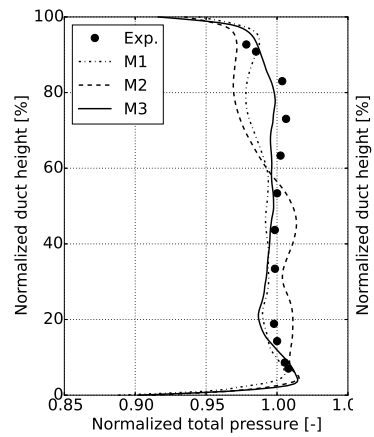

(a) Total pressure.

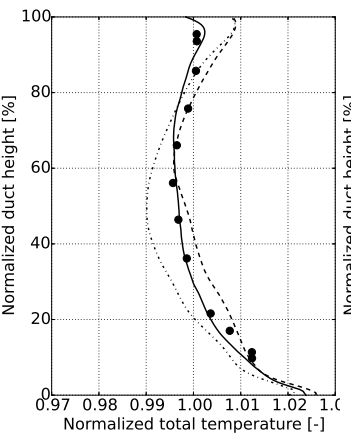

(b) Total temperature.

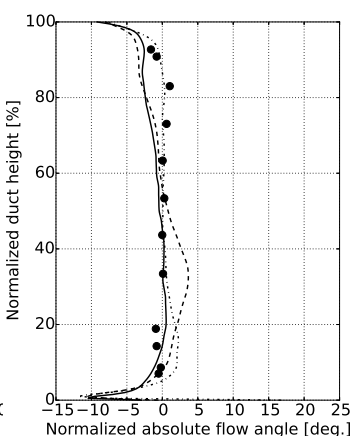

(c) Absolute flow angle.

Fig. 8 Radial distribution of time and azimuthally averaged quantities, at section 270 downstream of $\mathrm{S} 1$.

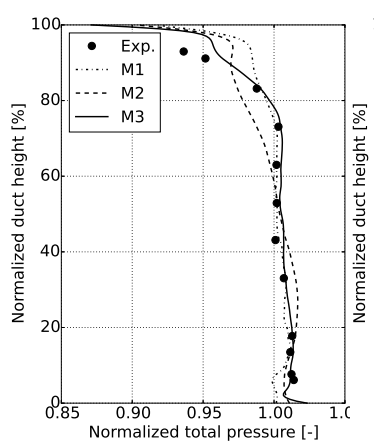

(a) Total pressure.

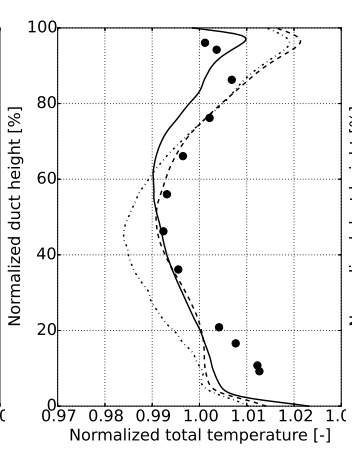

(b) Total temperature.

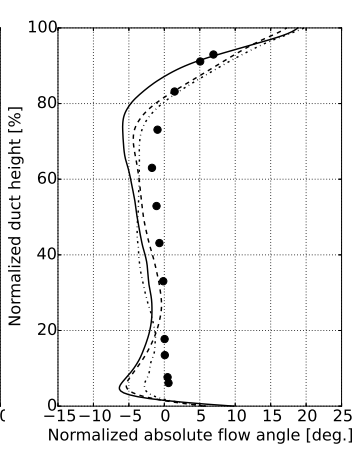

(c) Absolute flow angle.

Fig. 9 Radial distribution of time and azimuthally averaged quantities at section 27A downstream of R2.

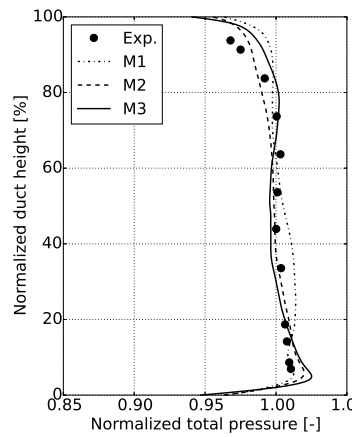

(a) Total pressure.

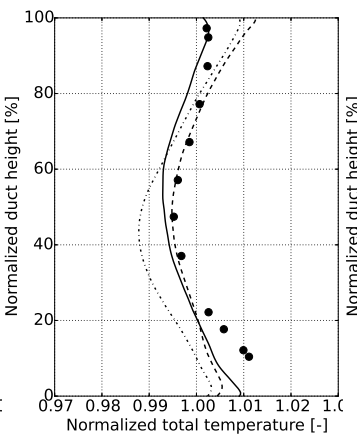

(b) Total temperature.

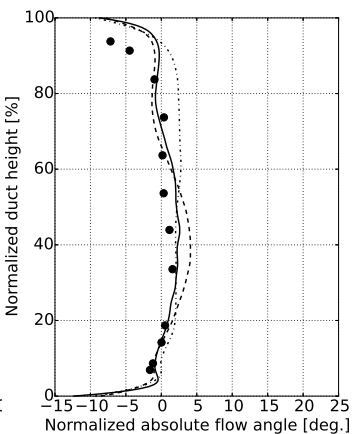

(c) Absolute flow angle.

Fig. 10 Radial distribution of time and azimuthally averaged quantities at section 280 downstream of S2. 


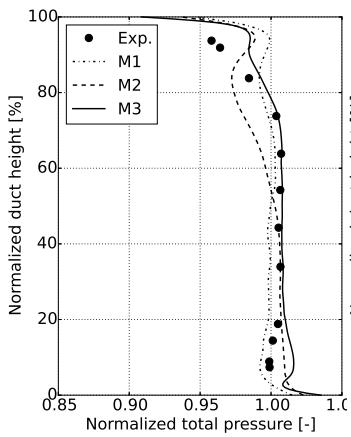

(a) Total pressure.

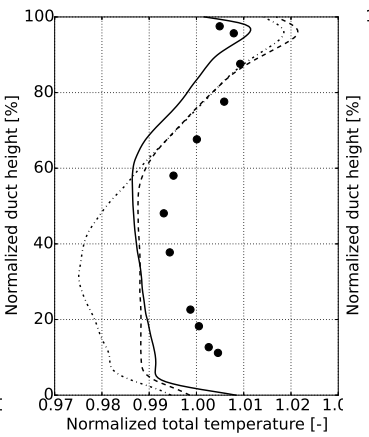

(b) Total temperature.

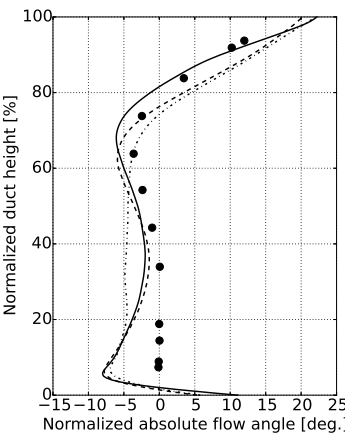

(c) Absolute flow angle.

Fig. 11 Radial distribution of time and azimuthally averaged quantities at section 28A downstream of R3.

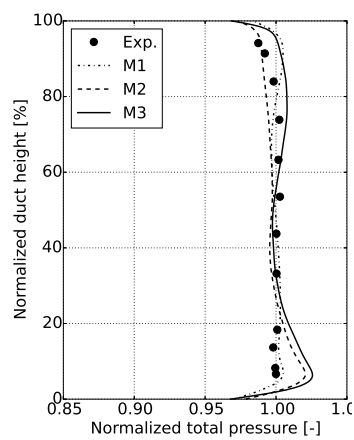

(a) Total pressure.

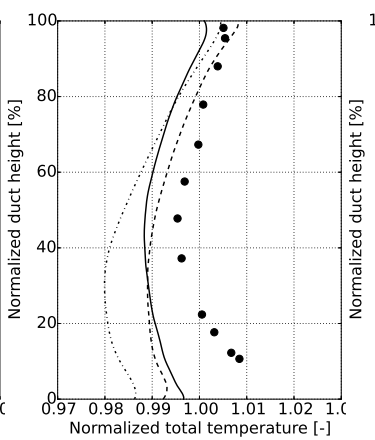

(b) Total temperature.

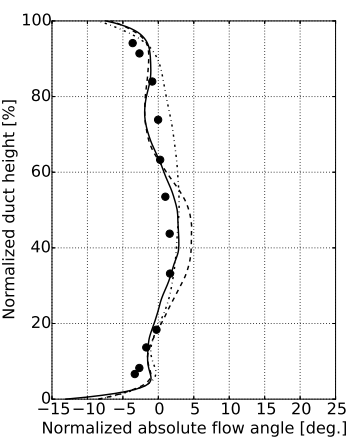

(c) Absolute flow angle.

Fig. 12 Radial distribution of time and azimuthally averaged quantities at section 290 downstream of S3.

For all the graphs (Figs. 7 to 12), the M3 grid generally provides results closer to experimental data than the coarse (M1) and the intermediate (M2) grids. This is for instance noticeable for total pressure in the upper part of the duct in each section. As explained in Sec. 2.2, the flow physics in this region is indeed very complex due to multiple interactions between rotor and stator wakes, tip leakage flows in the rotors and tip casing boundary layers. It thus requires the mesh refinement level of M3 to be correctly simulated. For the rest of the duct height, the three grids provide fair results with respect to measurements. Regarding total temperature, experimental shape and levels are correctly predicted by M3 up to section 280 , downstream stator 2 (Fig. 7 to 10 ). Total temperature is then slightly under-predicted by the simulations in the last stage. This could be due to the adiabatic condition at hub and casing walls that has more influence in the rear part of the machine. Nevertheless this shift is below $0.4 \%$ for M3. Absolute flow angles are predicted more accurately downstream stators than downstream rotors. The three grids tend indeed to under predict these angles downstream of rotors, in the 
main part of the duct. From Fig. 2, a too small value for $\alpha_{2}$ could originate from a too high value for $\beta_{2}$, indicating an under-turning by rotor blades possibly caused by too thick boundary layers predicted by LES. It could also come from a too high relative velocity $\mathbf{W}_{\mathbf{2}}$. A large difference can be observed in the lower part of the duct downstream R2 and R3, where simulations fail to predict the flow angle, whereas this is not the case downstream of R1. It is thus believed to be caused by the absence of the cavities below $\mathrm{S} 2$ and $\mathrm{S} 3$ in the simulation, that modify the flow in the main duct close to the hub. In the upper part of the duct (above $80 \%$ of height), the evolution of the angles strongly influenced by the tip leakage flow downstream each rotor are well reproduced in the simulations especially downstream of R2 and R3.

Globally, this comparison brings further evaluations to complement the global performance analysis. It indeed shows the need of proper grid refinement in the upper part of the duct, especially within and downstream rotor tip gaps, to improve the prediction of total pressure and temperature profiles. LES predictions for the three grids are nevertheless found to be less than few percent away from measurements. This is similar or better than existing URANS results [47].

\section{Flow analysis downstream rotating blade rows}

The analysis of radial profiles of total pressure in Sec. 4.2 highlights significant differences between the three grids and measurements downstream of each rotor, in the upper $20 \%$ of the duct height. A more detailed analysis is thus interesting, using the unsteady data available, for instance in section 26A. Figure 13 presents $2-\mathrm{D}$ maps of total pressure, axial and tangential velocities in this section. The experimental values originate from fast pressure probes and LDA measurements. These unsteady measurements, phase-averaged with the rotational speed, are then averaged in the rotor reference frame. The LES data, extracted at the same section, are also averaged in the rotor reference frame. In the test rig, measurements cannot be performed up to the hub and casing walls. The measured area is thus limited, as shown in figures related to experimental results. For LES, the whole area of section $26 \mathrm{~A}$ is represented, and two white lines indicate the limits of the measured data. Note that color scales are identical for all plots of a given variable.

Radial profiles of $P_{t}$ for M1 and M2 downstream of each rotor (Figs. 7(a), 9(a), 11(a)) exhibit a pressure peak close to the casing at about $95 \%$ of the normalized duct height. This feature is visible in Fig. 13(a) for M1 and M2, where local pockets of high total pressure are present between each rotor wake. These pockets are neither present in the experimental data nor in the M3 results, explaining the better prediction by the most refined grid. In M2 and M3, the rotor wakes identified by low $P_{t}$ values seem thinner than the measured ones. This could be a consequence of the measurement technique bringing blockage in the flow. Indeed when looking at LDA results coming from a non-intrusive technique (Figs. 13(b) and 13(c)), measured and simulated wakes seem to have similar widths. In the coarse grid M1, the axial velocity deficit within the rotor wakes is too large and is better predicted by M2 and M3. The effect of progressive grid refinement in the upper part of the duct is further evidenced in Fig. 13(b), since the zone of high axial velocity extends in the radial direction when going from M1 to M3, the latter being similar to LDA data. Concerning the tangential velocity (Fig. 13(c)), 


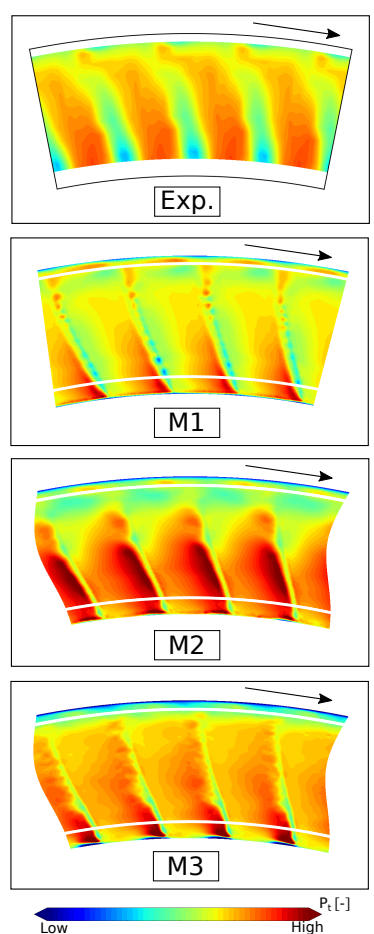

(a) Total pressure.
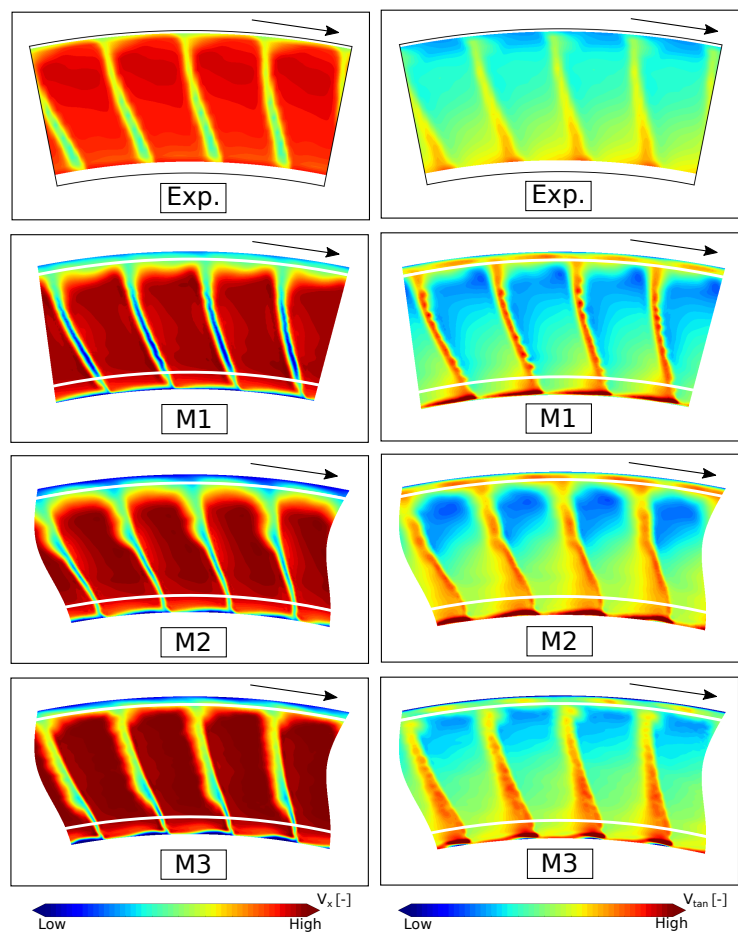

(b) Axial velocity.

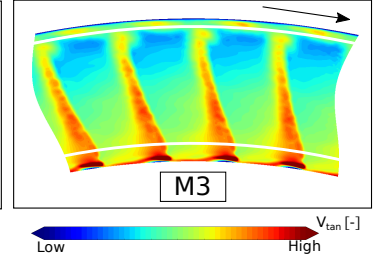

(c) Tangential velocity.

Fig. 13 Time-averaged quantities in the rotor reference frame, in section 26A downstream of R1. Arrows indicate the rotational direction.

pockets of high values are found in M1 and M2 close to the casing. They are directly related to the high total pressure pockets previously observed. Similar observations performed downstream of R3 indicate that a coarse mesh in the rotor tip region is responsible for an over-prediction of tangential velocity. Tangential velocity being $V_{2} \sin \left(\alpha_{2}\right)$ (see Fig. 2), the increase of absolute flow angle $\alpha_{2}$ is mostly caused by the too low values of axial velocity in Fig. 13(b) for M1 and M2. This could be the indication of too thick predicted casing boundary layers. This is consistent with the use of coarse grids in this region for M1 and M2.

As introduced in Sec. 2.2, tip leakage vortices in rotors represent a major source of losses. To evaluate the prediction of this critical flow feature, Fig. 14 presents the total pressure fluctuations downstream of each rotor, from section 26A (Fig. 14(a)) to section 28A (Fig. 14(c)). The experimental and numerical data are averaged in the rotor reference frame, and the fluctuations are computed relatively to the surface-averaged total pressure for each case. Looking at the experimental data, the rotor wakes are characterized by higher values of pressure fluctuations. Close to the casing, regions of high fluctuations are also visible. They correspond to the tip leakage vortex (see Fig. 3(b)), originating from one blade and migrating as well as mixing with the wake of the neighboring blade (from right to left in the figure). This vortex is smaller in size and lower in turbulence level in 26A than in 27A and 28A. The coarse (M1) grid tends to over-predict the size and the turbulence level 


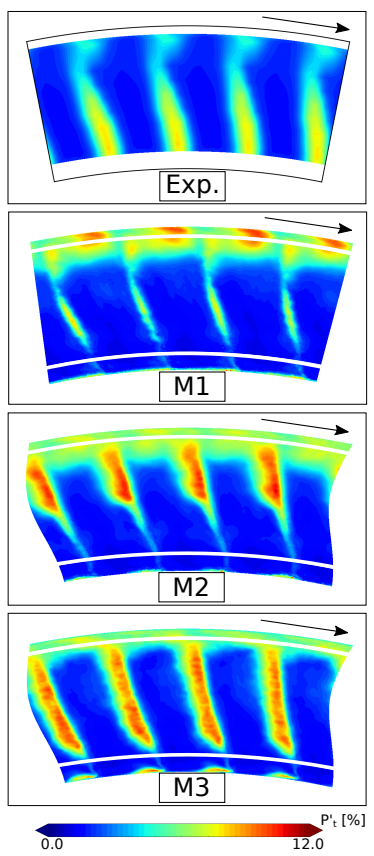

(a) Section 26A.

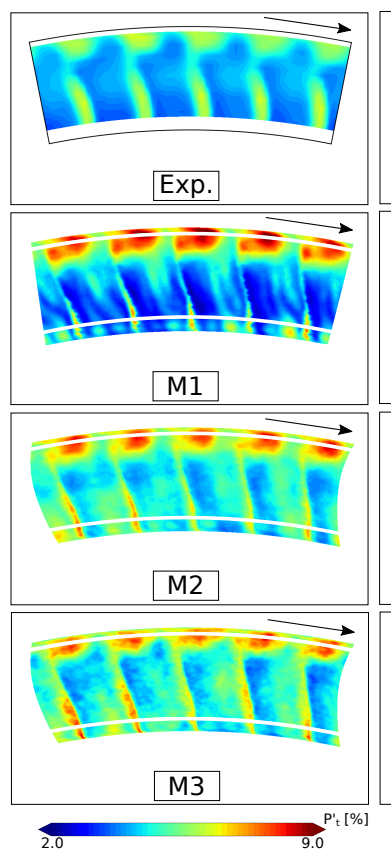

(b) Section 27A.

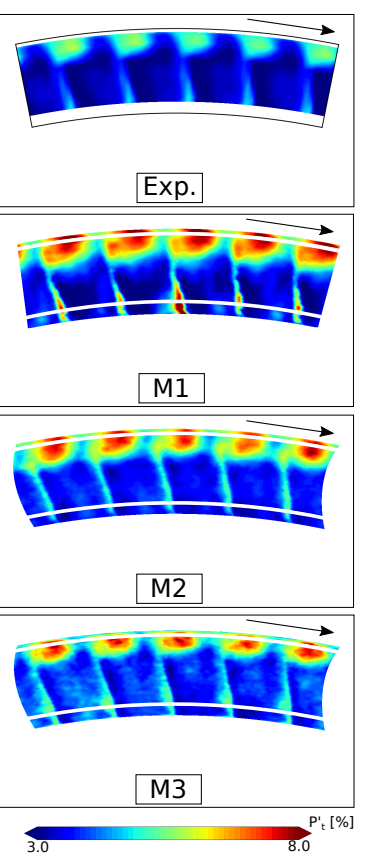

(c) Section $28 \mathrm{~A}$.

Fig. 14 Intensity of total pressure fluctuations, averaged in the rotor reference frame. Arrows indicate the rotational direction.

of tip leakage flows downstream of each rotor. The intermediate grid (M2) seems to predict a correct size for the tip leakage flows, but a too high level in terms of fluctuations. Finally the grid refinement in M3 is seen to allow getting the best prediction of the tip leakage flows among the three grids, both in terms of vortex size and amplitude of fluctuations.

The non-intrusive LDA technique also allows the evaluation of the tip leakage flow predictions. For instance, Fig. 15 shows axial and tangential velocity fluctuations in section 28A. The presence of the tip leakage flow is again highlighted by high velocity fluctuations, close to the casing on the pressure sides of the blades, as measured by LDA. When comparing experimental and LES data, successive coarsening of the grid resolution in the tip region is again shown to increase the tip leakage flows both in size and in amplitude.

\section{Tip-flow analysis in first rotor}

The flow in the tip region of high-pressure compressors is known to be critical, and the first rotor of CREATE has been particularly studied both experimentally [3] and numerically [44]. LDA allows measuring the flow within the rotor-blade channels as they are moving. Figures 16(a) and 17(a) present the axial velocity, averaged in the rotating reference frame, in terms of mean and RMS quantities. Measurements are performed at 4 axial positions along the blade, from $20 \%$ (position 1) to $91 \%$ (position 4) of axial chord from the blade leading edge. Data are 


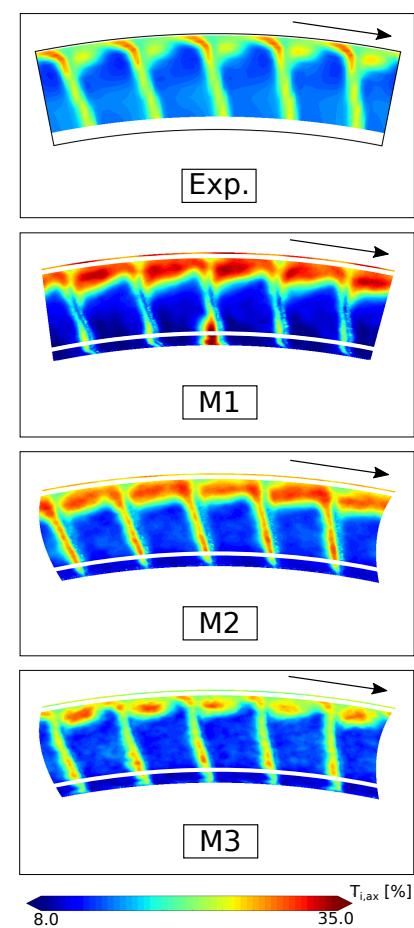

(a) Axial velocity.
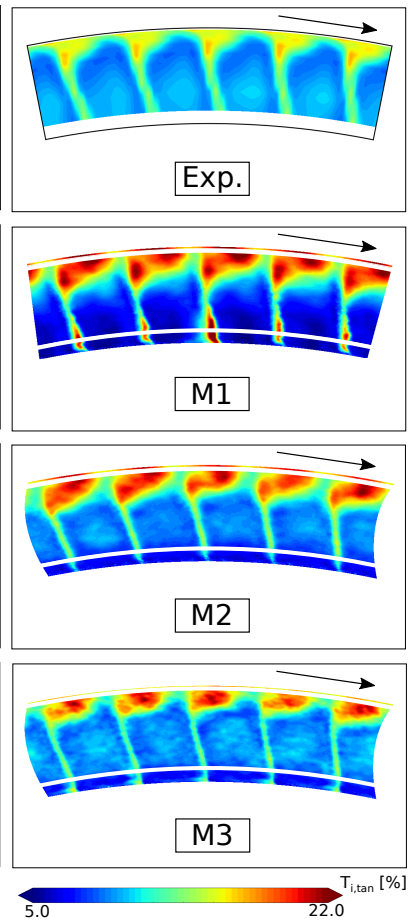

(b) Tangential velocity.

Fig. 15 Intensity of velocity fluctuations, averaged in the rotor reference frame, in section $28 \mathrm{~A}$ downstream of R3. Arrows indicate the rotational direction.

only available in the tip region, from $82 \%$ - $90 \%$ to $98 \%$ of duct height $\mathrm{h} / \mathrm{H}$. The blades are schematically represented in Figs. 16(a) and 17(a). Similar data, extracted from the three LES at the same positions and for the same radial extents, are added to Figs. 16 and 17 for comparisons. In Fig. 16, spatial variations of velocity are represented removing the surface-averaged value for each case. Color scale is again kept identical for all plots. In Fig. 17, color scale is identical for all the cases, the fluctuations being computed with respect to the local averaged velocity.

For M1 and M2, Fig. 16 highlights that the low velocity region close to the casing wall is larger than the measured one. This region creates a higher blockage, thus inducing a higher axial velocity at lower radii. This low velocity region is however correctly predicted by M3. After mid-chord (positions 3 and 4), the spatial variations of $V_{x}$ are particularly well reproduced by M3.

The second-order moment shown in Fig. 17 is consistent with the previous analysis. Indeed the region of high losses, represented by low axial velocity and high fluctuations, is over-predicted in size and amplitude with M1 and M2. M3 slightly over predicts the fluctuations, but their distributions are much closer to the experimental results.

A rotor tip flow can also be investigated through static pressure recorded at the wall. Figure 18(a) corresponds to the wall pressure recorded by high-frequency response pressure transducers, flush mounted within the tip casing. 24 transducers 


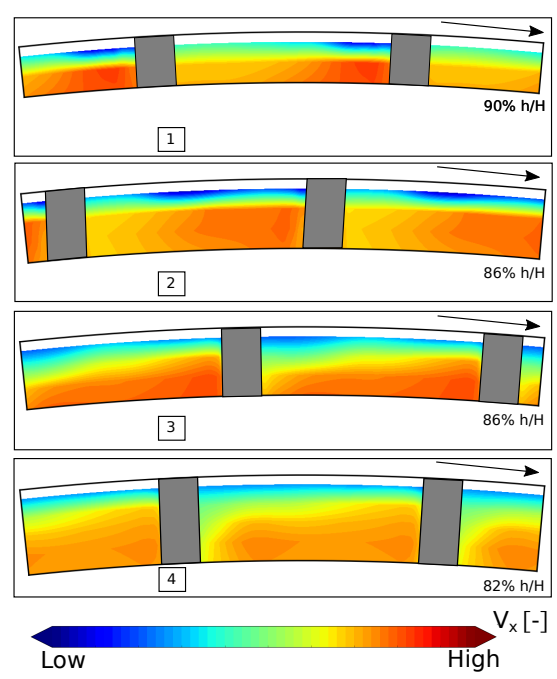

(a) Experiments.

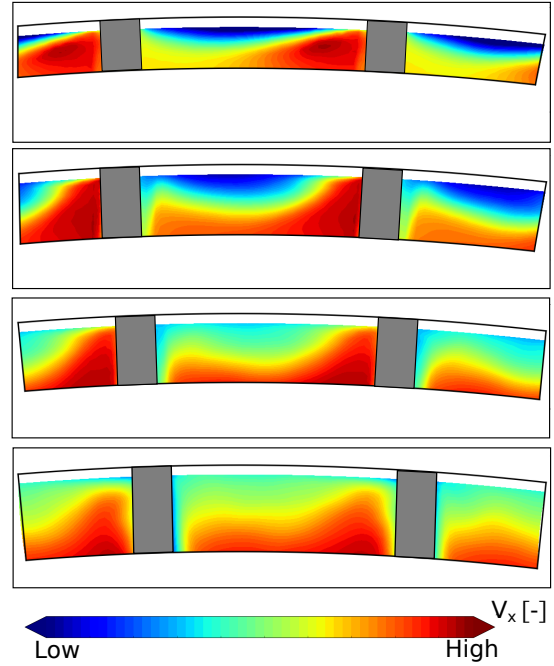

(c) LES M2.

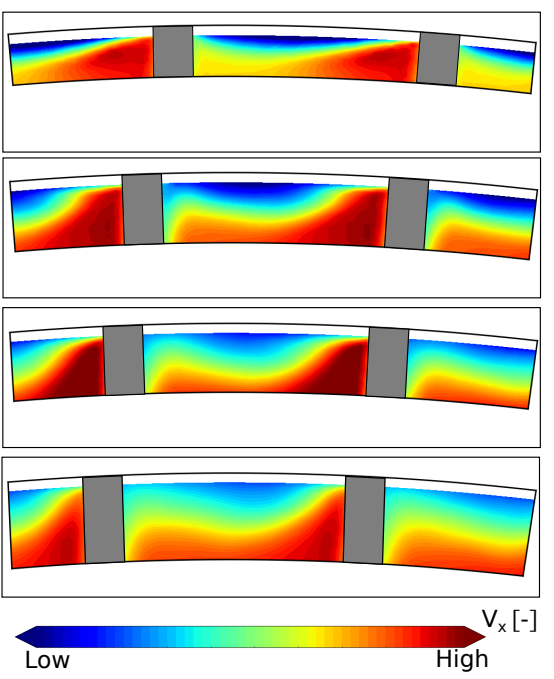

(b) LES M1.

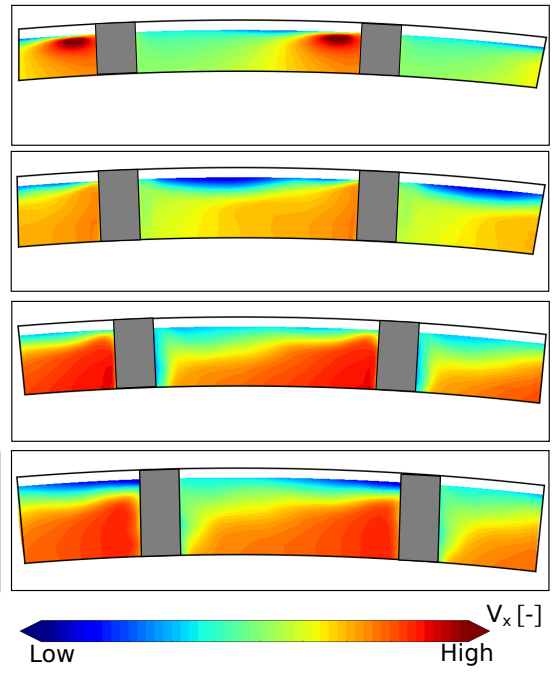

(d) LES M3.

Fig. 16 Normalized axial velocity, time averaged in the rotor reference frame: the spatial variations are shown without their local surface averaged value. Color scale is identical for all plots. Arrows indicate the rotational direction. Positions 1 to 4 correspond to $20 \%, 44 \%, 67 \%$ and $91 \%$ of axial chord respectively.

are used along the axial direction, and pressure is time-averaged in the rotating reference frame. The same data, extracted from the three LES, are added to Fig. 18. Only the spatial variations of pressure are shown, their mean value being removed for each case. Color scale is set to be identical for all plots. Sketches of the blade tip profiles are added to the figures for better understanding. The pressure gradient in each blade channel, from the pressure side of one blade to the suction side of the neighboring blade, is globally similar in experiments and LES. As noted 


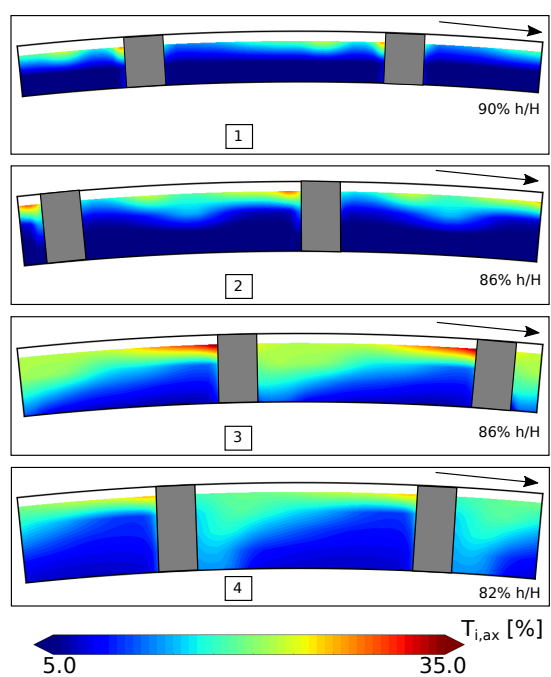

(a) Experiments.

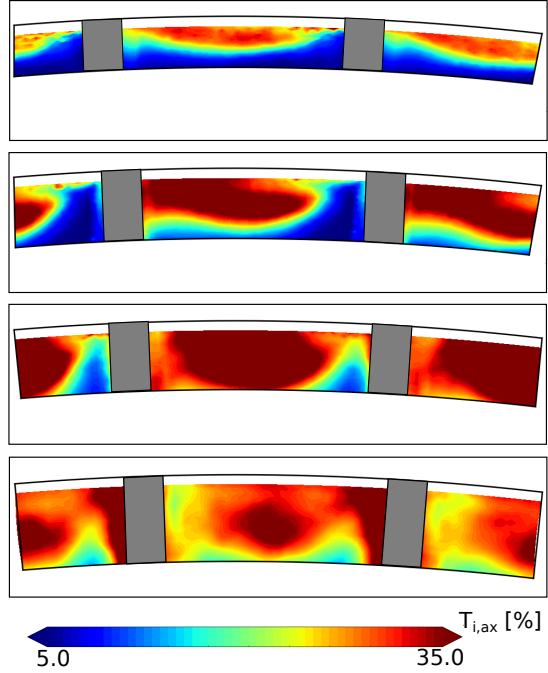

(c) LES M2.

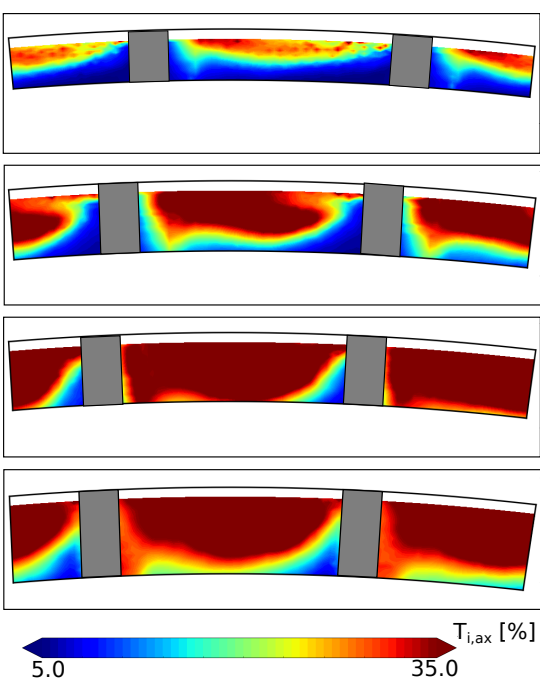

(b) LES M1.

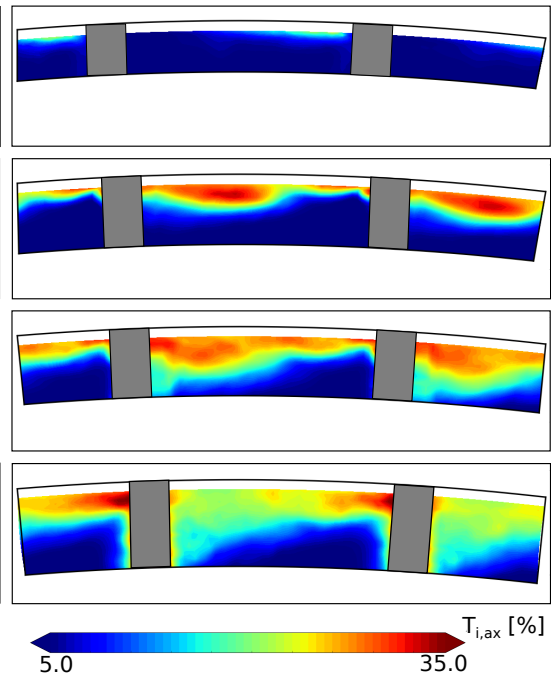

(d) LES M3.

Fig. 17 Intensity of axial velocity fluctuations, time averaged in the rotor reference frame. Color scale is identical for all plots. Arrows indicate the rotational direction. Positions 1 to 4 correspond to $20 \%, 44 \%, 67 \%$ and $91 \%$ of axial chord respectively.

in [3] and introduced in Sec. 2.2, Fig. 18(a) highlights two typical mechanisms of a rotor blade tip flow region. First the Tip Clearance Vortex (TCV) originates downstream of the blade leading edge, and develops within the blade channel along a straight line represented in the figures. The path of this vortex is correctly predicted by the three LES. However only M3 highlights a clearly defined path, in which the pressure evolution is similar to the measurements. Second, in 18(a), the Tip Separation Vortex (TSV) originates further away from the blade leading 


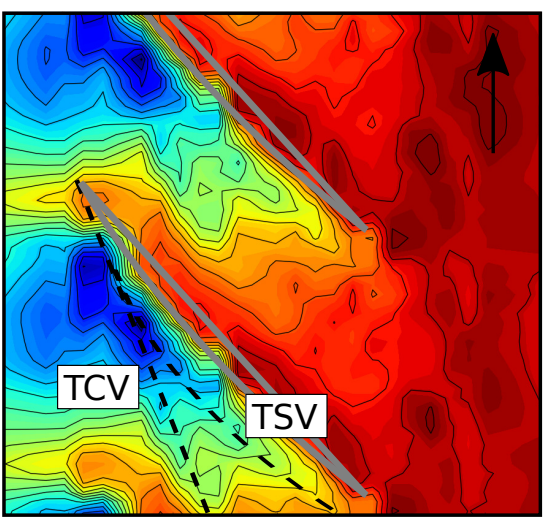

(a) Experiments.

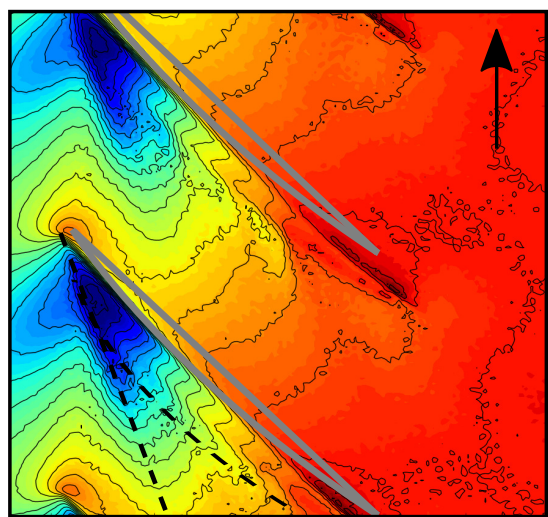

(c) LES M2.

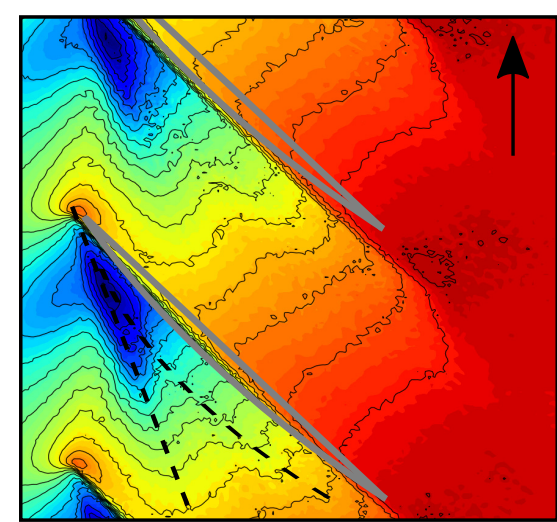

(b) LES M1.

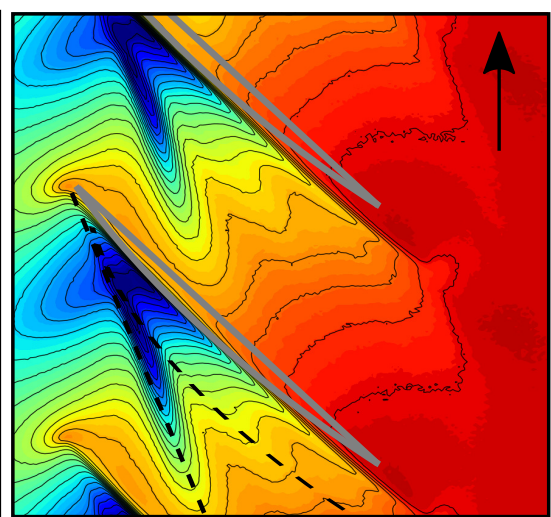

(d) LES M3.

Fig. 18 Spatial variations of static pressure on the casing wall above the tip of R1 blades, averaged in the rotor reference frame. Colorscale is identical for all plots, going from low (blue) to high (red) values. Arrows indicate the rotational direction.

edge. This vortex typically stays close to the blade suction side, as indicated by the curved dashed line representing its trajectory. For M1, the TSV path is not really identifiable. The grid seems indeed too coarse to capture this mechanism. The grid refinement in M2 allows capturing the TSV, however its extent seems smaller than in 18(a). It probably gets too dissipated by the grid. In M3, the path of the TSV is clearly defined, and its extent is very similar to the measurements. This shows the interest of progressive grid refinement, allowing to capture more and more precisely typical mechanisms occurring in the tip region of rotor blades. It can thus be expected that losses prediction gets improved as well in this critical zone.

The flow in the tip region of rotor 1 being validated for the LES grid M3, the analysis of associated three-dimensional vortical structures is performed in Fig. 19 on an instantaneous solution. Vortices are highlighted using the Q-criterion where Q stands for the second invariant of the velocity gradient tensor [20]. In Fig. 19(a) representing a view upstream of rotor blade tips, the tip clearance 


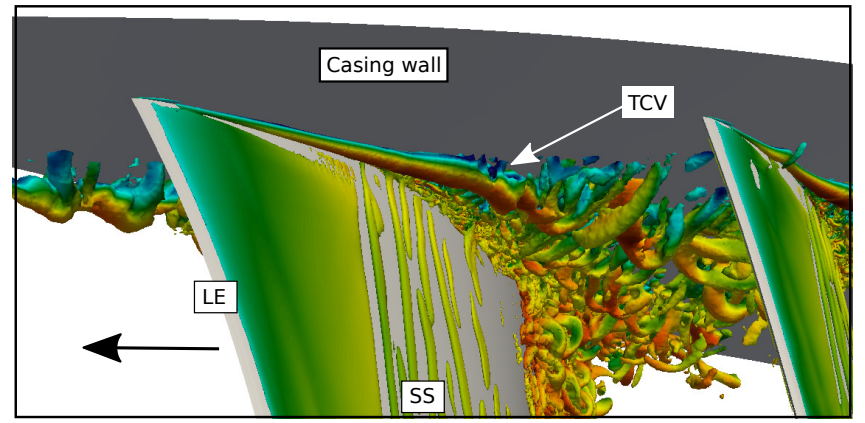

(a) View upstream of rotor 1.

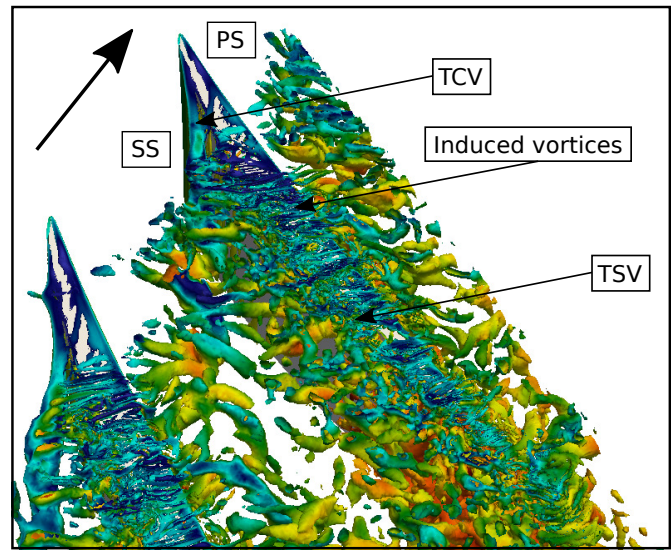

(b) View above rotor 1, casing wall being removed.

Fig. 19 Instantaneous Q-criterion iso-surface colored by Mach number within a blade passage of rotor 1 , close to the tip casing, obtained in grid M3. Black arrows indicate the rotational direction.

vortex (TCV) is clearly visible. As observed in Fig. 18, it is generated slightly downstream of the leading edge and develops along a straight line as a single tube. It grows regularly and then breaks down into a much larger vortex with an helicoidal shape. Figure 19(b) shows that this vortex continues to approximately follow a straight line and impact the pressure side (PS) of the adjacent blade. This figure also highlights other expected structures sketched in Fig. 3(b), namely the induced vortices and the tip separation vortex (TSV). Finally this analysis demonstrates the interest of such a refined simulation, that accurately predicts the flow in a critical region of an axial compressor and allows reaching detailed vortical structures.

\section{Analysis of losses}

From a global point of view and according to Denton [10], aerodynamic losses in a turbomachine result from entropy creation. The entropy variation between the inlet (index $i$ ) and a point $\mathbf{x}$ within the duct is: 

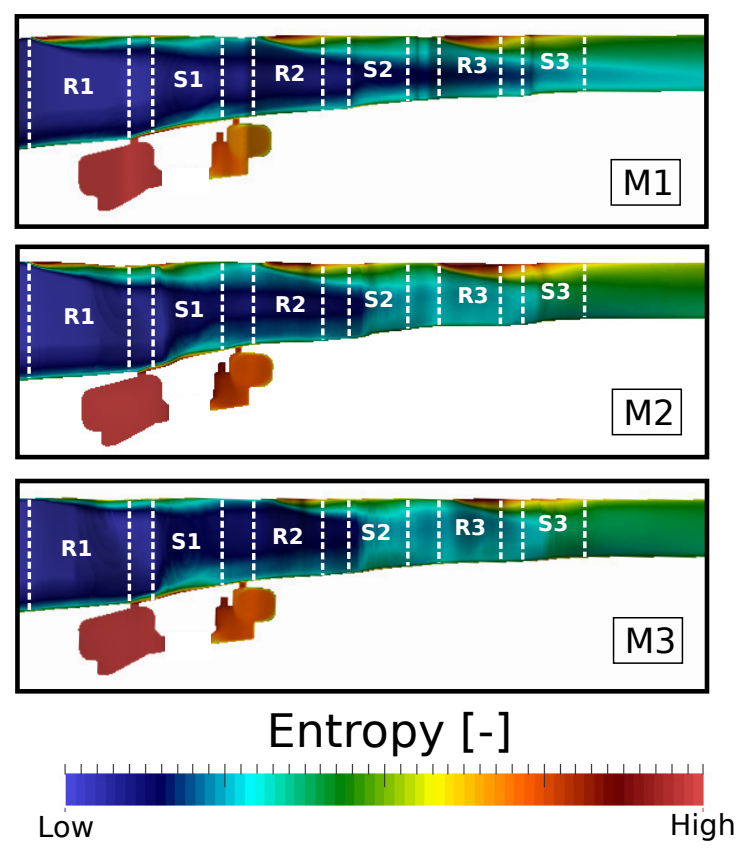

Fig. 20 Time-averaged entropy along the compressor, averaged in the azimuthal direction. Leading and trailing edges of blades are sketched with dashed white lines. Labyrinth seals have been removed from the figures.

$$
s(\mathbf{x})-s_{i}=C_{p} \ln \left(\frac{T(\mathbf{x})}{T_{i}}\right)-R \ln \left(\frac{P(\mathbf{x})}{P_{i}}\right)
$$

where $C_{p}$ and $R$ are specific heat capacity at constant pressure and gas constant respectively. The time-averaged entropy within the whole domain has been averaged along the circumferential direction and is represented in Fig. 20 for the three grids using the same color scale.

Several regions of entropy creation can be identified. The hub boundary layer presents similar entropy distribution among the grids. The cavity below stator 1 also corresponds to a region of high losses. The main differences among the three grids are located close to the tip casing. The losses generated at the tip of rotor blades are clearly visible. They increase in size and amplitude from upstream to downstream, showing that an upstream rotor enhances losses in a rotor located downstream. These regions of entropy creations are similar for M1 and M2, but smaller in size and amplitude for M3. This is related to the analyses performed in Secs. 5 and 6 highlighting too large predictions of tip leakage vortices for M1 and M2. A similar distribution of entropy within the main passage of the duct is noticeable in M2 and M3 grids, for instance in S2, R3 and S3, whereas losses are differently distributed in M1. This indicates a relevant grid resolution for M2 to correctly predict time-averaged losses within the main passage of the duct. 


\section{Conclusions}

This paper presents Wall-Modeled Large Eddy Simulations (WMLES) of an actual multistage high-pressure compressor. The features of the TurboAVBP numerical method required for such massive simulations have been demonstrated. The unstructured approach of the solver allows meshing complex geometries such as a recirculating cavity, proper rotor-stator interface treatments between each blade row and efficient parallel algorithms provide computations of more than one billion of degrees of freedom. Going beyond the feasibility demonstration, this study has evaluated the simulations using available experimental data, from integrated quantities to time-resolved measurements. Three grids of progressive refinement have been built to study the influence of mesh resolution on the results.

Thanks to their affordable cost, coarse (M1) and intermediate (M2) grids were simulated at several operating points. When comparing with experiments, these WMLES accurately predict the performance curve of the compressor; however only the intermediate grid is able to closely approach the last stable experimental point at low mass flow rate. Only a single operating point has been targeted for the finest grid (M3) because of its large computational cost. Comparisons of time and azimuthally averaged radial profiles with measurements show a clear improvement in flow prediction with M3 in the upper part of the duct. The need to account for recirculating cavities in the computational domain is also demonstrated since this appears necessary to accurately predict flow angle close to hub walls.

Using fast pressure probes and Laser Doppler Anemometry (LDA) time-resolved data, simulations have been evaluated downstream of rotors. Rotor wakes in the main stream are correctly simulated by M2 and M3. The coarse grid resolution close to the rotors tip casings for M1 and M2 provides too thick boundary layers that modify the flow in this critical region. Indeed using under-resolved grids in the rotor blade tip region yields too large leakage vortices in terms of size and amplitude. Aerodynamic losses are in this case over-estimated, as further evidenced looking at entropy creation in the whole compressor.

Focusing on the first rotor, it has been shown how the complex flow features in the blade tip region are accurately simulated in the finest grid M3. Indeed using LDA measurements within blade passages and data from fast pressure sensors mounted in the casing, the tip clearance vortex and the tip separation vortex are similar to the ones found in the simulation. These phenomena are known to drive performances and stability of axial compressors, thus the need for their accurate prediction.

Beyond the feasibility demonstration of WMLES of multistage compressors, this study indicates the required grid refinement according to the expected fidelity of the results. An intermediate grid indeed allows correctly reaching the performance curve at low mass flow rate as well as main flow features such as wakes and losses within the main passage of the duct. For a precise description in the rotor tip region, a grid refinement similar to M3 appears necessary. It then gives access to a large flow database than can be exploited for improving understanding of physical phenomena. In the future, the presented LES will be compared with state-of-the-art RANS and URANS data, to provide recommendations on the use of WMLES for compressor design. 
Acknowledgements The authors acknowledge the financial support of SAFRAN within the Numerical Core 2020 project. This work was granted access to the HPC resources of CINES under the allocation x20162a6074 made by GENCI. The authors are grateful to X. Ottavy and J. Schreiber (LMFA) for fruitful discussions on CREATE.

\section{References}

1. AVBP: http://cerfacs.fr/avbp7x/

2. Cagnone, J.S., Rasquin, M., Hillewaert, K., Hiernaux, S.: Large eddy simulation of a low pressure compressor cascade at high incidence. In: 12th European Turbomachinery Conference, ETC2017-318 (2017)

3. Courtiade, N.: Experimental analysis of the unsteady flow and instabilities in a high-speed multistage compressor. Ph.D. thesis, Ecole Centrale Lyon (2012)

4. Courtiade, N., Ottavy, X.: Experimental study of surge precursors in a high-speed multistage compressor. J. Turbomach. 135 (2013)

5. Courtiade, N., Ottavy, X., Gourdain, N.: Modal decomposition for the analysis of the rotor-stator interactions in multi-stage compressors. J. Th. Sc. 21(3), 276-285 (2012)

6. Crevel, F., Gourdain, N., Moreau, S.: Numerical simulation of aerodynamic instabilities in a multistage high-speed high-pressure compressor on its test-rig. part I: Rotating stall. J. Turbomach. 136 (2014)

7. Crevel, F., Gourdain, N., Ottavy, X.: Numerical simulation of aerodynamic instabilities in a multistage high-speed high-pressure compressor on its test-rig. part II: Deep surge. J. Turbomach. 136 (2014)

8. Cumpsty, N.A.: Compressor aerodynamics. Krieger Publishing Company (2004)

9. Denos, R., Paniagua, G. (eds.): Aero-engine design: from state-of-the-art turbofans towards innovative architectures. Lecture Series, Von Karman Institute for Fluid Dynamics (2008)

10. Denton, J.D.: Loss mechanisms in turbomachines. In: ASME International Gas Turbine and Aeroengine Congress and Exposition, 93-GT-435 (1993)

11. Duchaine, F., Dombard, J., Gicquel, L., Koupper, C.: On the importance of inlet boundary conditions for aerothermal predictions of turbine stages with large eddy simulation. Computers \& Fluids 154, 60-73 (2017)

12. Duchaine, F., Jauré, S., Poitou, D., Quémerais, E., Staffelbach, G., Morel, T., Gicquel, L.: Analysis of high performance conjugate heat transfer with the openpalm coupler. Computational Science \& Discovery 8(1), 15003-15021 (2015)

13. Gicquel, L., Gourdain, N., Boussuge, J.F., Deniau, H., Staffelbach, G., Wolf, P., Poinsot, T.: High performance parallel computing of flows in complex geometries. Comptes Rendus Mecanique 339(2-3), $104-124$ (2011)

14. Gourdain, N.: Prediction of the unsteady turbulent flow in an axial compressor stage. part 1: Comparison of unsteady RANS and LES with experiments. Computers \& Fluids 106, 119-129 (2015)

15. Gourdain, N., Gicquel, L., Collado, E.: Comparison of RANS and LES for prediction of wall heat transfer in a highly loaded turbine guide vane. J. Prop. Power 28, 423-433 (2012)

16. Gourdain, N., Sicot, F., Duchaine, F., Gicquel, L.: Large eddy simulation of flows in industrial compressors: a path from 2015 to 2035. Phil. Trans. R. Soc. Lond. A 372 (2014)

17. Gourdain, N., Wlassow, F., Ottavy, X.: Effect of tip clearance dimensions and control of unsteady flows in a multi-stage high-pressure compressor. J. Turbomach. 134 (2012)

18. Granet, V., Vermorel, O., Leonard, T., Gicquel, L., , Poinsot, T.: Comparison of nonreflecting outlet boundary conditions for compressible solvers on unstructured grids. AIAA Journal 48(10), 2348-2364 (2010)

19. Hah, C.: Large eddy simulation of transonic flow field in NASA Rotor 37. Tech. Rep. NASA/TM-2009-215627, NASA John Glenn Research Center (2009)

20. Hunt, J.C.R., Wray, A.A., Moin, P.: Eddies, streams, and convergence zones in turbulent flows. In: Proc. of the Summer Program , pp. 193-208. Center for Turbulence Research, NASA Ames/Stanford Univ. (1988)

21. Joo, J., Medic, G., Philips, D.A., Bose, S.T.: Large eddy simulation of a compressor rotor. In: Proceedings of the Summer Program. Center for Turbulence Research (2014) 
22. Koupper, C., Poinsot, T., Gicquel, L., Duchaine, F.: Compatibility of characteristic boundary conditions with radial equilibrium in turbomachinery simulations. AIAA Journal 52(12), 2829-2839 (2014)

23. de Laborderie, J., Duchaine, F., Gicquel, L.: Analysis of a high-pressure multistage axial compressor at off-design conditions with coarse Large Eddy Simulations. In: 12th European Turbomachinery Conference, ETC2017-125 (2017)

24. de Laborderie, J., Duchaine, F., Gicquel, L., Vermorel, O., Wang, G., Moreau, S.: Numerical analysis of a high-order unstructured overset grid method for compressible LES of turbomachinery. J. Comput. Phys. 363, 371-398 (2018)

25. de Laborderie, J., Duchaine, F., Vermorel, O., Gicquel, L., Moreau, S.: Application of an overset grid method to the Large Eddy Simulation of a high-speed multistage axial compressor. In: ASME Turbo Expo, GT2016-56344 (2016)

26. de Laborderie, J., Moreau, S., Berry, A.: Compressor stage broadband noise prediction using a large-eddy simulation and comparisons with a cascade response model. In: 19th AIAA/CEAS Aeroacoustics Conference (2013)

27. Lax, P.D., Wendroff, B.: Difference schemes for hyperbolic equations with high order of accuracy. Commun. Pure Appl. Math. 17, 381-398 (1964)

28. Leonard, T., Sanjose, M., Moreau, S., Duchaine, F.: Large eddy simulation of a scale-model turbofan for fan noise source diagnostic. In: 22nd AIAA/CEAS Aeroacoustics Conference (2016)

29. Mailach, R., Vogeler, K.: Aerodynamic blade row interactions in an axial compressor Part I: unsteady boundary layer development. J. Turbomach. 126 (2004)

30. McMullan, W., Page, G.: Towards large eddy simulation of gas turbine compressors. Prog. in Astronautics and Aeronautics 52(0), $30-47$ (2012)

31. Mersinligil, M., Brouckaert, J.F., Courtiade, N., Ottavy, X.: A high temperature high bandwith fast response total pressure probe for measurements in a multistage axial compressor. J. Eng. Gas Turb. and Power 134 (2012)

32. Mersinligil, M., Brouckaert, J.F., Courtiade, N., Ottavy, X.: On using fast response pressure sensors in aerodynamic probes to measure total temperature and entropy generation in turbomachinery blade rows. J. Eng. Gas Turb. and Power 135 (2013)

33. Monier, J.F., Boudet, J., Caro, J., Shao, L.: Budget analysis of turbulent kinetic energy in a tip-leakage flow of a single blade: RANS vs zonal LES. In: 12th European Turbomachinery Conference, ETC2017-113 (2017)

34. Moureau, V., Lartigue, G., Sommerer, Y., Angelberger, C., Colin, O., Poinsot, T.: Numerical methods for unsteady compressible multi-component reacting flows on fixed and moving grids. J. Comput. Phys. 202(2), 710-736 (2005)

35. Mouret, G.: Adaptation of phase-lagged boundary conditions to large-eddy simulation in turbomachinery configuration. Ph.D. thesis, INP Toulouse (2016)

36. Mouret, G., Gourdain, N., Castillon, L.: Adaptation of phase-lagged boundary conditions to large-eddy simulation in turbomachinery configurations. In: ASME Turbo Expo, GT2015-42516. Montreal, Canada (2015)

37. Odier, N., Duchaine, F., Gicquel, L., Dufour, G., Rosa, N.G.: Comparison of LES and RANS predictions with experimental results of the fan of a turbofan. In: 12th European Turbomachinery Conference, ETC2017-126 (2017)

38. Ottavy, X., Courtiade, N., Gourdain, N.: Experimental and computational methods for flow investigation in high-speed multistage compressor. J. Prop. Power 28(6), 1141-1155 (2012)

39. Papadogiannis, D., Duchaine, F., Gicquel, L., Wang, G., Moreau, S.: Effects of subgrid scale modeling on the deterministic and stochastic turbulent energetic distributions in Large-Eddy Simulations of a high-pressure turbine stage. J. Turbomach. 138, 091005091005-10 (2016)

40. Papadogiannis, D., Duchaine, F., Gicquel, L., Wang, G., Moreau, S., Nicoud, F.: Assessment of the indirect combustion noise generated in a transonic high-pressure turbine stage. J. Eng. Gas Turb. and Power 138(4), 0415030 (8 pp.) (2015)

41. Piomelli, U.: Wall-layer models for large-eddy simulations. Prog. Aerospace Sci. 44(6), 437-446 (2008). DOI 10.1016/j.paerosci.2008.06.001

42. Poinsot, T., Lele, S.: Boundary conditions for direct simulations of compressible viscous flows. J. Comput. Phys. 101(1), 104-129 (1992). DOI 10.1016/0021-9991(92)90046-2

43. Pope, S.B.: Turbulent flows. Cambridge University Press (2000)

44. Riera, W., Castillon, L., Marty, J., Leboeuf, F.: Inlet condition effects on the tip clearance flow with zonal detached eddy simulation. J. Turbomach. 136 (2014) 
45. Sagaut, P.: Large eddy simulation for incompressible flows. Springer (2002)

46. Schonfeld, T., Rudgyard, M.: Steady and unsteady flows simulations using the hybrid flow solver AVBP. AIAA Journal 37(11), 1378-1385 (1999)

47. Schreiber, J.: Investigation of experimental and numerical methods, and analysis of stator clocking and instabilities in a high-speed compressor. Ph.D. thesis, Ecole Centrale Lyon (2016)

48. Schreiber, J., Ottavy, X., Boum, G.N., Gourdain, N.: Influence of rotor tip flow field misprediction on rotating disturbance near surge in a high speed multistage compressor. In: ASME Turbo Expo, GT2016-57372 (2016)

49. Scillitoe, A.D., Tucker, P.G., Adami, P.: Numerical investigation of three-dimensional separation in an axial flow compressor: the influence of freestream turbulence intensity and endwall boundary layer state. J. Turbomach. 139 (2017)

50. Smagorinsky, J.: General circulation experiments with the primitive equations: 1 . the basic experiment. Mon. Weather Rev. 91, 99-164 (1963)

51. Teramoto, S.: Large eddy simulation of a transonic compressor cascade with boundary layer transition. In: 41st AIAA/ASME/SAE/ASEE Joint Propulsion Conference \& Exhibit. 10 - 13 July 2005, Tucson, Arizona. Tucson, AZ, USA (2005)

52. Tucker, P.: Computation of unsteady turbomachinery flows: Part 2 LES and hybrids. Prog. Aerospace Sci. 47(7), 546 - 569 (2011)

53. Tyacke, J., Tucker, P., Loveday, R., Vadlamani, N., Watson, R., Naqavi, I., Yang, X.: Large eddy simulation for turbines: Methodologies, cost and future outlooks. J. Turbomach. 136(6), 061009 (2013). DOI 10.1115/1.4025589

54. Wang, G., Duchaine, F., Papadogiannis, D., Duran, I., Moreau, S., Gicquel, L.Y.M.: An overset grid method for large eddy simulation of turbomachinery stages. J. Comput. Phys. 274, 333-355 (2014)

55. Wang, G., Moreau, S., Duchaine, F., de Laborderie, J., Gicquel, L.: LES investigation of aerodynamics performance in an axial compressor stage. In: 22nd Conference of the CFD Society of Canada. Toronto, Canada (2014)

56. Wang, G., Sanjose, M., Moreau, S., Papadogiannis, D., Duchaine, F., Gicquel, L.: Noise mechanisms in a transonic high-pressure turbine stage. Int. J. Aeroacoust. 15, 144-161 (2016)

57. Watson, R.A., Tucker, P.G., Wang, Z.N., Yuan, X.: Towards robust unstructured turbomachinery large eddy simulation. Computers \& Fluids 118, 245-254 (2015) 